\title{
Spatial expression of Hox cluster genes in the ontogeny of a sea urchin
}

\author{
César Arenas-Mena*1,2, Andrew R. Cameron ${ }^{1}$ and Eric H. Davidson ${ }^{1}$ \\ ${ }^{1}$ Division of Biology 156-29, California Institute of Technology, Pasadena, CA 91125, USA \\ 2Stowers Institute for Medical Research, Kansas City, MO 64110, USA \\ *Author for correspondence (e-mail: arenas@its.caltech.edu) \\ Accepted 17 August; published on WWW 9 October 2000
}

\section{SUMMARY}

The Hox cluster of the sea urchin Strongylocentrous purpuratus contains ten genes in a $500 \mathrm{~kb}$ span of the genome. Only two of these genes are expressed during embryogenesis, while all of eight genes tested are expressed during development of the adult body plan in the larval stage. We report the spatial expression during larval development of the five 'posterior' genes of the cluster: SpHox7, SpHox8, SpHox9/10, SpHox11/13a and SpHox11/13b. The five genes exhibit a dynamic, largely mesodermal program of expression. Only $\mathrm{SpHox} 7$ displays extensive expression within the pentameral rudiment itself. A spatially sequential and colinear arrangement of

\section{INTRODUCTION}

The Hox gene cluster is a pan-bilaterian developmental patterning device, but thus far experimental evidence on its use refers almost entirely to chordates and arthropods. These are all direct developing animals that display a twofold symmetry around the adult anterior/posterior $(\mathrm{A} / \mathrm{P})$ axis. The single $500 \mathrm{~kb}$ Hox gene cluster of the sea urchin Strongylocentrous purpuratus was recently cloned (Martinez et al., 1999) and the complete sequence of the ten-gene complex will soon be available (Cameron et al., 2000). Unlike chordates and arthropods, S. purpuratus is a pentamerally symmetrical animal that uses a process of maximal indirect development, such that the ciliated free-living larva to which the embryo gives rise bears virtually no resemblance to the adult echinoid body plan. This emerges from a complex developmental progression, much of which takes place in an imaginal rudiment growing within the lumen of the feeding larva. The embryo and the larva are bilaterally symmetrical in their general organization, and the fivefold radially symmetrical structure that is a definitive phyletic character of modern adult echinoderms is generated within the rudiment (Pearse and Cameron, 1991; Davidson et al., 1998; Peterson et al., 1997, 2000a). Many of the larval structures contribute no descendants to the adult body plan; for instance, most of the larval oral and aboral ectoderm, the pharynx, and the distal part of the intestine. All of those larval tissues are jettisoned during metamorphosis (Pearse and Cameron, 1991). We have previously shown that except for two of the Hox genes, viz. SpHox7 and SpHoxl1/13b, the Hox gene cluster of expression domains is found in the somatocoels, the paired posterior mesodermal structures that will become the adult perivisceral coeloms. No such sequential expression pattern is observed in endodermal, epidermal or neural tissues of either the larva or the presumptive juvenile sea urchin. The spatial expression patterns of the Hox genes illuminate the evolutionary process by which the pentameral echinoderm body plan emerged from a bilateral ancestor.

Key words: Hox, Strongylocentrous purpuratus, Sea urchin, Gene expression
$S$. purpuratus is not used at all to build the structures of the embryo, that is, to generate the basic feeding larva, while all of the eight Hox genes studied are copiously transcribed during the process of adult body plan formation, In the tissues that will give rise to the adult (Arenas-Mena et al., 1998). Here we describe the spatial expression of the five 'posterior' Hox genes during the early to mid stages of adult body plan function, the first observations of their kind on an indirect-developing, radially organized animal.

Molecular phylogeny confirms unequivocally the classically held supposition (Metschnikoff, 1881; Hyman, 1955) that echinoderms are the sister group of the hemichordates within the deuterostomes; the chordates are thus the sister group of the hemichordate plus echinoderm clade (Cameron et al., 2000; Wada and Satoh, 1994; Bromhan and Degnan, 1999). But while echinoderms are radially symmetrical, hemichordates (and chordates) and virtually all protostomes are bilaterally symmetrical. The radial symmetry of the echinoderms is therefore a derived feature, and the ancestor they shared with the hemichordates was bilaterally symmetrical. The initial bilateral arrangement of hemichordate (enteropneust) and echinoderm larval coeloms at early postembryonic stages is in fact remarkably similar (Peterson et al., 1997). Both groups essentially form three sets of coeloms: in echinoderms the anterior pair of coeloms is called the axocoels; the middle coeloms are the hydrocoels; and the posterior coeloms are the somatocoels. Consistent with expectation from its $5^{\prime}$ position in the S. purpuratus Hox gene cluster, we found that the SpHox11/13b gene is expressed exclusively in the somatocoels (Peterson et al., 2000a). This observation, combined with 
paleontological evidence and current interpretations of skeletal structure in echinoderms (Mooi and David, 1997) enabled us to propose a reconstruction of the evolutionary process by which the echinoderm body plan was derived from its bilateral ancestral form (Peterson et al., 2000a). The fivefold symmetry of the echinoderm body first appears within a structure including the left hydrocoel, but the axis of fivefold symmetry is orthogonal to the original $\mathrm{A} / \mathrm{P}$ axis (i.e. as if we had five arms positioned radially around our $\mathrm{A} / \mathrm{P}$ axis). These changes in axial symmetry lead to a shift in the position of the coeloms so that they stack under one another. Thus, moving inward from the end of the body plan that derives from the original anterior end, still the site of the mouth, the sequence of coelomic derivatives is the left hydrocoel and left somatocoel, then the right somatocoel. Specific predictions as to Hox gene use follow from this interpretation, given that an ancestral function of the Hox gene cluster in bilaterians is $\mathrm{A} / \mathrm{P}$ pattern specification, colinear with the gene order.

To examine Hox gene expression within the largely unexplored anatomy of the developing rudiment, we had to modify procedures for in situ hybridization and then reconstruct the patterns of gene use from serial sections. Owing to limitations of space, we are able to present only a small fraction of these in what follows. But while the results are indeed consistent with the evolutionary pathway proposed by Peterson et al. (2000a), many surprises nonetheless lay in wait for us: for the five Hox genes considered here we found that expression was largely mesodermal; we observed an unexpected sequence of Hox gene expression patterns within the somatocoel at cross orientation to the adult A/P axis; and we saw a remarkable number of apparent co-options of Hox gene use in developing structures that are special features of echinoderms.

\section{MATERIALS AND METHODS}

\section{Culture of larvae}

Sea urchin larvae were grown as described previously (Cameron et al., 1989). Cultures were kept at $16^{\circ} \mathrm{C}$ with constant stirring in filtered sea water at a density of 200 larvae/l. Feeding was provided 5 days after fertilization with Rodomonas lens at about 3000 cells per ml.

\section{Whole-mount in situ hybridization}

Animals were fixed in $4 \%$ formaldehyde, 0.1 M MOPS ( $\mathrm{pH} 7$ ), $0.5 \mathrm{M}$ $\mathrm{NaCl}$ for 1 hour at room temperature for later stages, defined as having several adult spines (as those in Fig. 3D-F). For earlier stages with pentameral rudiments (such as those in Fig. 2) a solution with both $2 \%$ paraformaldehyde and $2 \%$ formaldehyde was used in order to improve morphological preservation, despite some loss of hybridization signal intensity. After five washes in at least 10 volumes of $0.1 \mathrm{M}$ MOPS, $0.5 \mathrm{M} \mathrm{NaCl}$ and $0.1 \%$ Tween-20 (MOPS buffer), the samples were stored indefinitely in $70 \%$ ethanol at $-20^{\circ} \mathrm{C}$. Rehydration was accomplished with three washes of 15 minutes each, in at least 10 volumes of MOPS buffer in $1.7 \mathrm{ml}$ tubes. Hybridization was conducted in a solution consisting of $70 \%$ deionized formamide, $0.5 \mathrm{M} \mathrm{NaCl}, 0.1 \mathrm{M}$ MOPS ( $\mathrm{pH}$ 7), $1 \mathrm{mg} / \mathrm{ml} \mathrm{BSA} \mathrm{(solubilized} \mathrm{in} \mathrm{water}$ first) and $0.1 \%$ Tween-20. Two conditioning transfers to fresh hybridization buffer preceded a 3 hour prehybridization at $50^{\circ} \mathrm{C}$. Riboprobes containing digoxigenin-UTP were synthesized by conventional methods; for the specific Hox gene probes used see Arenas-Mena et al. (1998). It was found advantageous to employ a one week long hybridization period, using $0.1 \mathrm{ng} / \mu \mathrm{l}$ of riboprobe at $50^{\circ} \mathrm{C}$ (in the above hybridization buffer). After hybridization, samples were washed five times in MOPS buffer at room temperature to remove the probe, incubated for an additional 3 hours under hybridization conditions and washed three more times in MOPS buffer. The samples were blocked with $10 \mathrm{mg} / \mathrm{ml} \mathrm{BSA} \mathrm{in} \mathrm{MOPS}$ buffer for 20 minutes at room temperature and then with $10 \%$ goat serum plus $1 \mathrm{mg} / \mathrm{ml} \mathrm{BSA}$ at $37^{\circ} \mathrm{C}$ for 30 minutes in MOPS buffer. Incubation with a $1 / 1500$ dilution of the alkaline phosphatase conjugated Fab fragments (Roche Molecular Biochemicals, Indianapolis, IN) was performed overnight at room temperature. The antibody was removed with five washes in MOPS buffer over an interval greater than 12 hours. After two washes in alkaline phosphatase buffer for a total of an hour, the reactions were developed by conventional methods with NBT/BCIP. Addition of $10 \%$ dimethyl formamide to the alkaline phosphatase development buffer greatly enhanced the staining reaction. The reaction was stopped by dilution in MOPS buffer.

\section{Embedding, sectioning, viewing and photography}

After the staining reaction, the specimens were embedded in Durkupan water-soluble medium for optical microscopy (Fluka, Milwaukee, WI). Serial sections of approximately $7.5 \mu \mathrm{m}$ were obtained. Images of the sections were made with a Roche Camera system using Wimcam and Photoshop software.

\section{RESULTS}

\section{Stages of rudiment development and in situ hybridization}

The derivation of the coelomic constituents of the imaginal rudiment from the embryonic mesodermal territories has been summarized earlier (Davidson et al., 1998; for morphological description of echinoid rudiment development see Pearse and Cameron, 1991; Hyman, 1955; Von Ubisch, 1913; MacBride, 1903). The main structural features of the developing larva that are relevant to our present purposes are illustrated in Fig. 1. In Fig. 1A the disposition of the larval coelomic sacs can be seen after about two weeks of larval development. The rudiment is beginning to form on the left-hand side of the stomach. Here the left hydrocoel (h) has assumed a thickened form, and it confronts the invaginating oral ectodermal pouch called the vestibule (v). These tissues soon unite and from their apposition many of the major oral structures of the adult body plan will derive. These include the radially organized water vascular and central nervous systems. The anterior coeloms or axocoels are also indicated in Fig. 1A, on the right-hand side indistinct from the hydrocoel $(\mathrm{ax}-\mathrm{h})$. Below the hydrocoels are the thin sheet-like somatocoelar sacs (s, in Fig. 1A), extending on both sides over the stomach. By homology with hemichordates, and by virtue of the observation that the $5^{\prime}$ most gene SpHox11/13b is expressed in the somatocoels, we identify the somatocoels as posterior structures (Peterson et al., 2000a). By the stage shown in Fig. 1B,C, the rudiment is more fully developed and the coelomic organization much more complex. Somatocoelar elements have grown up under the rudiment and are now interdigitated with hydrocoelar components, as illustrated in the following, and below this the somatocoels now extend widely around the stomach and intestine. Viewed face on from the left-hand side, as in Fig. 1C, the pentameral symmetry of the rudiment is clearly visible, in the five primary podia ( $\mathrm{pp}$ ) that can be seen protruding from it. In the center the adult mouth (am) will form: note that the oral 
Fig. 1. Larval anatomy of Strongylocentrous purpuratus. Live larvae were anesthetized in 3\% ethanol and viewed with differential interference contrast optics. (A) Part of a early eightarm larva about 10 days after the onset of feeding, viewed frontally. The oral ectoderm (oec) is towards the top and the aboral towards the bottom. The mouth (m), pharynx (ph) and stomach (st) are clearly visible. The anus is out of focus towards the objective. The original coelomic evaginations have extended along the gut and are now divided into three regions. On the larva left-hand side (L) the hydrocoel (h), or middle coelom, is bounded on its anterior by the axocoel (ax) and its posterior by the somatocoel (s). These remain as flattened sacs, while the hydrocoel is spherical. On the righthand $(\mathrm{R})$ side, the boundary between the axocoel and hydrocoel is indistinct (axh). The somatocoel can be distinguished by its position along the side of the stomach. The right axocoel-hydrocoel complex will form the dorsal vesicle, and part of the axial complex of the adult. The somatocoels will extend posteriorly to the midline. The invagination from the oral ectoderm termed the vestibule (v) can be seen. It will contact the left hydrocoel, and from the apposition of these tissues will form the primordium of the adult oral surface and its associated structures, i.e. the 'urchin rudiment'. (B) Eight-arm larva in the same orientation as (A), about three weeks old. The developing rudiment is clearly evident in this view. Projections of the hydrocoel have extended into the vestibule and together with the vestibular epithelium form the prominent primary podia (pp). The ciliated epaulets (ep) of the larval epithelium are now quite pronounced and the larval arms (lar) are fully extended by skeletal rods. A coalescence of blastocoelar cells that will form one of the adult plates, and its associated projections, lies below the epaulet on the right side of the larva (red arrow). (C) Left lateral view of an eight-arm larva of the same age as (B). The adult mouth (am; red arrowhead) will form in the center of the pentameral urchin rudiment. This has now grown to occupy a large region of the larval body alongside the stomach, which lies beneath the rudiment in this view. The pentameral organization of the rudiment is clearly evident. (D) The axial designations adopted for the sea urchin larvae used in this paper. (E) A schematic diagram of a larva in frontal view, similar to the photograph above the oral-aboral axis and the left-right axis are shown. (F) A schematic diagram in side view, similar to the photograph above. The oral-aboral axis and the anal-abanal axis are shown. Other abbreviations: a, anus; cb, ciliated band; ec, ectoderm; h, left hydrocoel; int, intestine; spi, larval spicule. Positional labels are circled. surface of the adult body faces orthogonally to the embryonic and larval oral surface and mouth $(\mathrm{m})$, i.e. towards the viewer, when compared with toward the top of the page.

When raised in the laboratory, $S$. purpuratus larvae develop at somewhat variable rates over the interval from the onset of feeding until competence to undergo metamorphosis is attained. Stages of larval development are therefore given here on the basis of anatomical progress rather than timing. Throughout this period the larval body per se undergoes much less change than does the rudiment, which grows from a few hundred cells to about 150,000 (Cameron et al., 1989). A stage usually attained by three weeks after fertilization in laboratoryraised S. purpuratus (Cameron et al., 1989) is the formation of a hydrocoelar torus, when pentameral symmetry first becomes evident. The most useful metric for subsequent stages of development is the number of inter-radial spines that have formed within the rudiment. Since the patterns of Hox gene expression are dynamic, it was very important to be able to compare results obtained at equivalent stages of rudiment development.

Single-stranded antisense RNA probes were obtained from the five genes so far identified at the 'posterior' end of the Hox cluster, i.e. from the 5' end, viz. SpHox11/13b, SpHox 11/13a, SpHox9/10, SpHox8 and SpHox7 (Arenas-Mena et al., 1998; Martinez et al., 1999). These were used for whole-mount in situ hybridization (WMISH) at all stages from embryogenesis through larval metamorphosis. After in situ hybridization, staining patterns in the larvae were examined in serial sections. Extensive alterations in procedures for WMISH were required for the larval samples, as detailed in Materials and Methods. Results obtained on the embryonic samples (not shown) merely confirmed what had been known earlier: only SpHox11/13b (Dobias et al., 1996) and SpHox7 (Angerer et al., 1989) are expressed during embryogenesis, largely as described. 


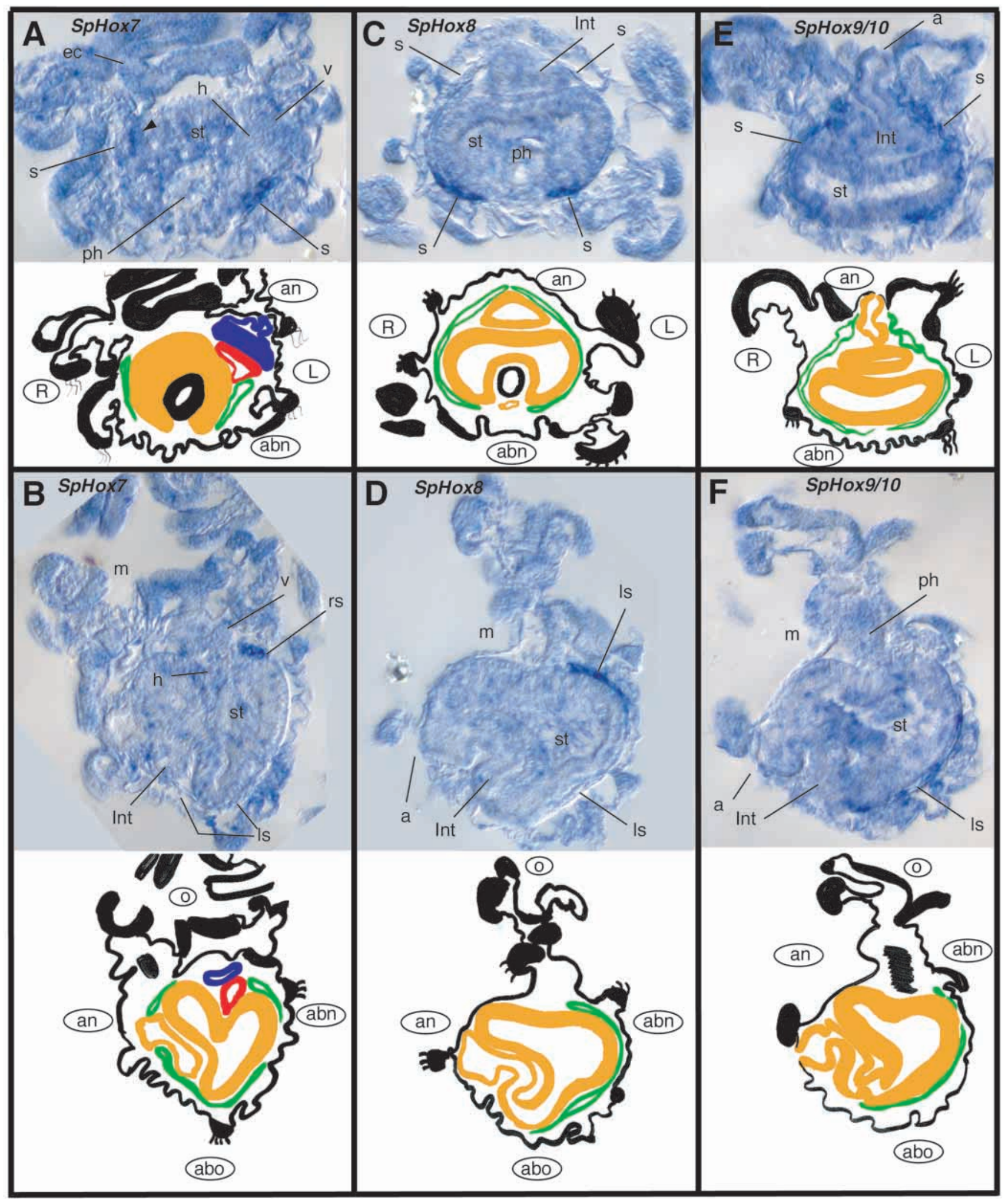

Fig. 2. Hox gene expression in larval somatocoels. Representative sections of larvae on which WMISH had been performed are shown (see Materials and Methods), using the indicated probes. The larvae have rudiments that start to acquire pentameral organization of their hydrocoels. Beneath each stained section (A-J) is a diagram illustrating the anatomy. The planes of the sections, with respect to the morphology of the larvae as a whole, are shown in the drawings $(\mathrm{K}-\mathrm{M})$, and the color code used in the anatomical diagrams is given in $(\mathrm{N})$. Hybridization of each 


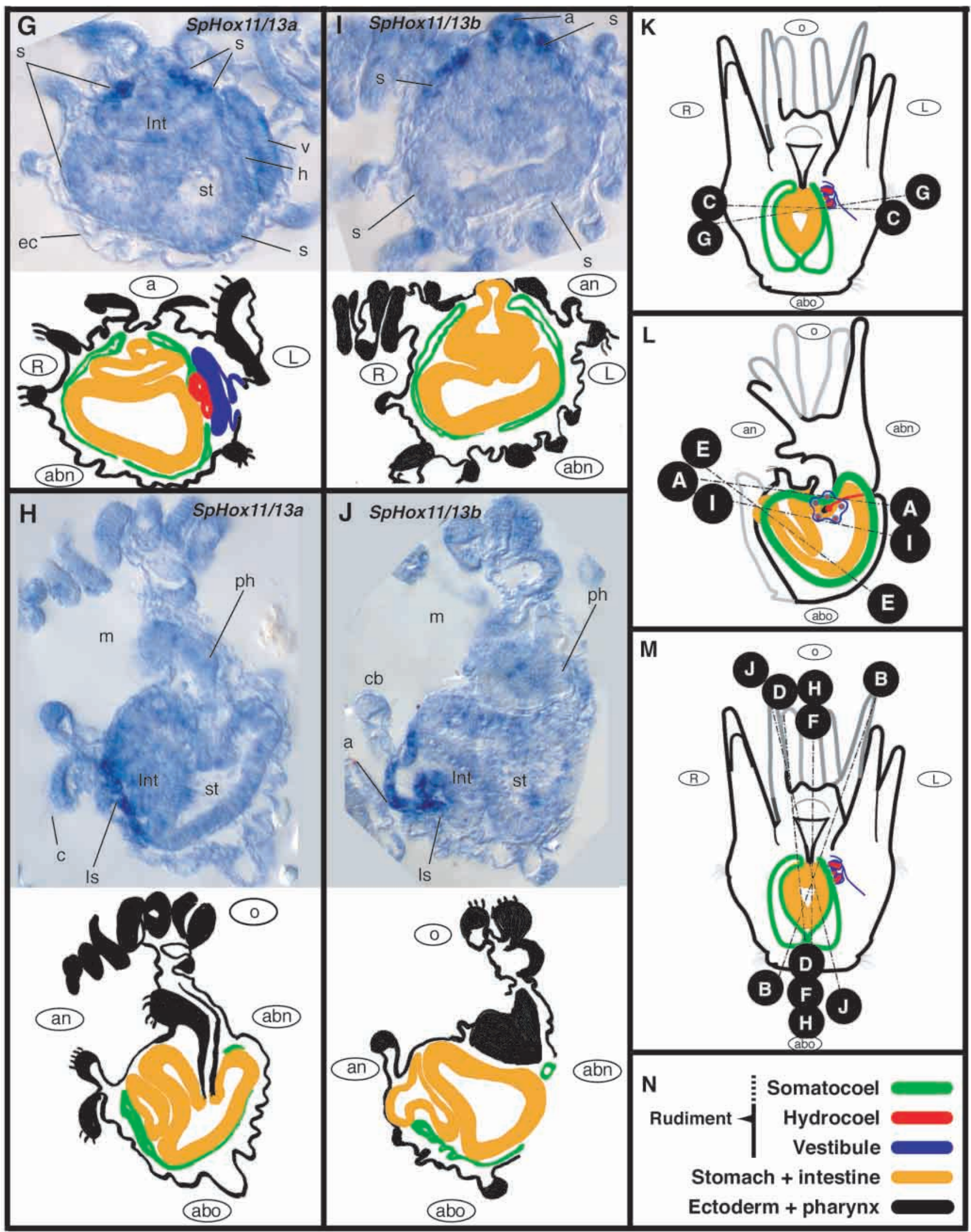

Hox gene is shown in both a transverse (top), and a saggital (below) section: $\operatorname{SpHox7}$ (A,B); SpHox8 (C,D); $\operatorname{SpHox9/10~(E,F);~SpHox11/13a~}$ (G,H); SpHox11/13b (I,J). Abbreviations are as in Fig. 1, except abn, abanal; abo, aboral; an, anal; ls, left somatocoel; ; o, oral; rs, right somatocoel. 
Table 1. Figures illustrating expression domains for posterior Hox genes in S. purpuratus larvae

\begin{tabular}{|c|c|c|c|c|c|}
\hline & SpHox7 & SpHox8 & SpHox $9 / 10$ & SpHox11/13a & SpHox11/13b \\
\hline Somatocoel & $2 \mathrm{~A}, \mathrm{~B}, 4 \mathrm{C}$ & $2 \mathrm{C}, \mathrm{D}, 3 \mathrm{~A}, 4 \mathrm{~A}$ & 2E,F, 3B,D & $2 \mathrm{G}, \mathrm{H}, 3 \mathrm{E}$ & 2I,J, 3C,F \\
\hline Intestine/anus & & & & & 2I,J \\
\hline Juvenile spines and associated structures & & & & $5 \mathrm{~B}$ & $3 \mathrm{~F}, 5 \mathrm{~A}, \mathrm{C}, \mathrm{F}$ \\
\hline Aboral ectoderm (vertex) & $4 \mathrm{C}, 6 \mathrm{~B}$ & & & & \\
\hline Mesenchyme & $6 \mathrm{~B}, \mathrm{D}, \mathrm{F}, \mathrm{H}$ & & & & \\
\hline Vestibular folds & $6 \mathrm{E}, \mathrm{F}, \mathrm{H}$ & & & & \\
\hline Floor of epineural canal (nerves) & $6 \mathrm{~F}, \mathrm{H}$ & & & & \\
\hline
\end{tabular}

Furthermore, the larval WMISH results are entirely consistent with the quantitative probe excess hybridization measurements of Arenas-Mena et al. (1998), using exactly the same probes; thus, it can be excluded that very high levels of transcript in just a few embryonic cells could have accounted for any of the results of those measurements. The WMISH patterns seen in metamorphosing animals were similar to those of advanced rudiment stages, and, with one exception, are not shown separately here.

As a guide to the WMISH results, Table 1 lists the locations in the larva of the observed domains of Hox gene expression, and the Figs in which these are illustrated. Each of the genes is expressed in a unique pattern, but these patterns share one important feature: all five genes are expressed in the somatocoels.

\section{Somatocoelar expression patterns}

The domains of expression of all five Hox genes in the somatocoels of late torus-stage larvae (i.e. slightly earlier than shown in Fig. 1B,C) are illustrated in Fig. 2. As an aid in interpreting the complex and unfamiliar anatomy of these larvae, color-coded drawings that represent the main features of the sections are shown beneath each. In general, the colored regions in these drawings are tissues that will contribute to the adult body plan (except for the anal area of the hindgut), while the black regions are jettisoned at metamorphosis (except for some local patches of the aboral ectoderm where arise adult test plates and spines). The diagrams in Fig. 2K-M are in the same orientation as the pictures in Fig. 1B,C. Note that the digestive tract is curved so that the intestine opens near the mouth (Figs 2L and 1C). The somatocoels, the main focus of Fig. 2, are in green. The expression patterns are shown in two aspects for each gene: in transverse sections in the top row of Fig. 2 (i.e. A,C,E,G,I) and in more or less saggital sections in the bottom row (i.e. B,D,F,H.J). The planes of section are indicated in the diagrams (K-M).

SpHox7 was expressed only in the regions of the somatocoel nearest the larval mouth, at the opposite side from the anus and behind the pharynx (Fig. 2A, staining at s on larval left side). Expression is symmetrical, but the section is oblique and the equivalent region of the right somatocoel is not included. In Fig. 2B, note that the stained region is confined to the top of the somatocoel; as the diagram in Fig. 2M shows, the equivalent region on the right-hand side is again not included in the section. Other sites of $S p H o x 7$ expression (e.g. arrowhead in Fig. 2A) are considered below.

SpHox 8 was expressed at the same stage in an abanal somatocoelar domain that overlaps that of SpHox7, but extended more towards the oral/aboral (O/Abo) midline of the larva, as viewed from either side. The domains of expression of this gene are shown in the transverse and saggital sections of Fig. 2C,D. The expression extended in the oral direction right up to the margin of the somatocoel.

SpHox $9 / 10$ was expressed the most broadly in the somatocoels of any of the five genes. As shown in Fig. 2E, its expression extended most of the way around the somatocoelar circumference, excluding only the anal and abanal regions at the oral side, and in the saggital section of Fig. $2 \mathrm{~F}$ it can be seen to reach all the way down to the aboral end of the somatocoel (only the left somatocoel is included in this image).

SpHox11/13a was expressed towards the anal side of the somatocoels, along the intestine (Fig. 2G). Its domain of expression extends up but does not include the region overlain by the imaginal rudiment (red and blue in the diagram). In the saggital section (Fig. $2 \mathrm{H}$ ), the expression of SpHox $11 / 13 \mathrm{a}$ can be seen to extend below the anal area all the way to the aboral vertex.

SpHox 11/13b was expressed exclusively around the anus. The section shown in Fig. 2I passes through the anus, and the somatocoels can be followed from there all the way around to the opposite side; only the anal region is stained. The sections in Fig. 2I,J transect the intestine and anus, which can be seen to express the gene together with the adjacent somatocoelar tissue. SpHox 1 1/13b begins to be expressed in the hindgut of the embryo, and this expression continues in the larva after feeding begins (Dobias et al., 1996; C. A.-M., A. R. C. and E. H. D., unpublished). Expression in the adjacent somatocoelar tissues is seen only after several days of larval development. For all the genes studied there was a general decrease in the intensity of the stain towards the aboral pole. This is shown by the broader area detected when using fixations that provide more intense staining (see Materials and Methods).

Fig. 2 provides a very interesting general result: the patterns of expression of the five Hox genes formed a sequential series, but surprisingly the sequence extended bilaterally, more or less cross-wise in the larva with respect to its O/Abo axis. That is, the most $5^{\prime}$ of the genes, SpHox 11/13b, was expressed in the anal area, and the more 'anterior' genes were expressed progressively towards the abanal side of the somatocoels, particularly at the top or oral side of the somatocoels (cf. diagrams in Fig. 7). An interpretation of this result is considered in the Discussion; but before proceeding thereto it is necessary to consider two additional parameters that progressively affect the somatocoelar expression pattern. This is a growing left-right asymmetry in the expression patterns, and other changes in somatocoelar expression patterns occur as development of adjacent structures proceeds.

In Fig. 3 the strong correlation is illustrated for several of the genes between expression levels and proximity of the rudiment. The first example is $\mathrm{SpHox} 8$ (Fig. 3A) where a strong 

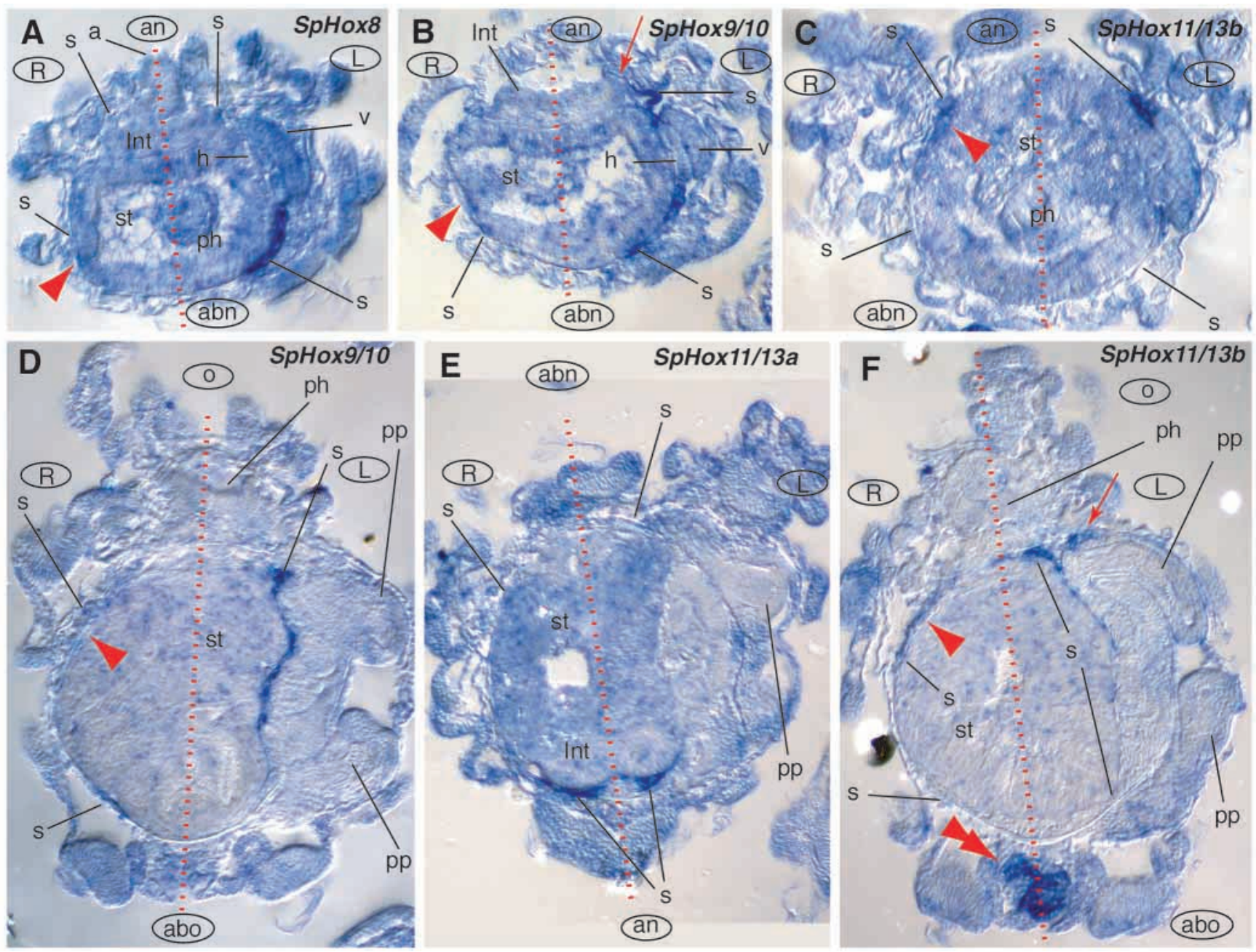

Fig. 3. Hox gene expression in the somatocoel is enhanced near the rudiment. (A-C) Transverse sections at the level of the rudiment at an early torus stage (see Fig. 2G for diagram of a similar section); (D-F), sections of more advanced stages, older than that in Fig. 1B. Broken red lines indicate the approximate plane of bilateral symmetry of the larval body. Red arrowheads indicate regions of the right somatocoel that are symmetrically located with respect to the regions of the left somatocoel that are stained. (A) $\mathrm{SpHox} 8$ expression near the rudiment, and in contrast, lack of expression in the corresponding region at the right side (red arrowhead). (B) SpHox9/10 expression is enhanced around the rudiment. The somatocoel has not yet extended between the hydrocoel and the stomach, thus at this stage there can be no signal from the somatocoel beneath the rudiment. (C) Expression of $5 p H o x 11 / 13 \mathrm{~b}$ in the immediate region of the rudiment, diminished at the equivalent region of the right somatocoel. In the anal region this same animal displays a perfectly symmetrical pattern as shown in the section of Fig. 2I.

(D) Frontal section; SpHox9/10 expression remains stronger in the left somatocoel at a more advanced stage; the somatocoel has now grown beneath the rudiment. (E) Transverse section; SpHox11/13a expression in the somatocoel remains symmetrical at later stages, as it was during earlier stages (see Fig. 2G). (F) Frontal section; SpHox11/13b expression continues at an enhanced level at the later stage; expression on the right side is detectable but at lower level (arrowhead). There is also expression in the margin of the vestibule (arrow) and in a structure at the aboral vertex (double arrowhead). See legends to Fig. 1 and 2 for abbreviations.

patch of somatocoelar expression was seen in the immediate vicinity of the rudiment, on the oral side. A striking asymmetry was generated (red arrowhead), which was entirely absent in sections below the level of the rudiment as seen in Fig. 2C, where Hox8 expression appeared symmetrical. Hox9/10 was also expressed asymmetrically at the torus stage, displaying enhanced activity near the rudiment (Fig. 3B). Later, at the stage when several adult spines have formed, the somatocoel has grown beneath the rudiment, and the specific region of the somatocoelar tissue underlying the hydrocoelar layer now displays a high level of expression, compared with the equivalent region on the right-hand side of the larva. This is illustrated in Fig. 3D (red arrowhead). But the same is not true of all the somatocoelar Hox gene expression patterns. Fig. 3E shows that Hoxl1/13a continued to be expressed in a perfectly symmetrical way, irrespective of the adjacent rudiment on the larval left side. Like SpHox7, SpHox8 and SpHox9/10, $\mathrm{SpHox11/13b}$ was also expressed asymmetrically as well, beginning at the torus stage (Fig. 3C) and continuing at the stage shown in Fig. 3F, which is similar to that of the larvae shown in Fig. 3D. It is interesting to note that the expression of this gene, which began precociously (in the endoderm) during embryogenesis, was no longer detectable in the anal region at the most advanced larval stages (not shown).

Somatocoelar expression of two of the Hox genes was also enhanced in the region directly adjacent to the stone canal, a 


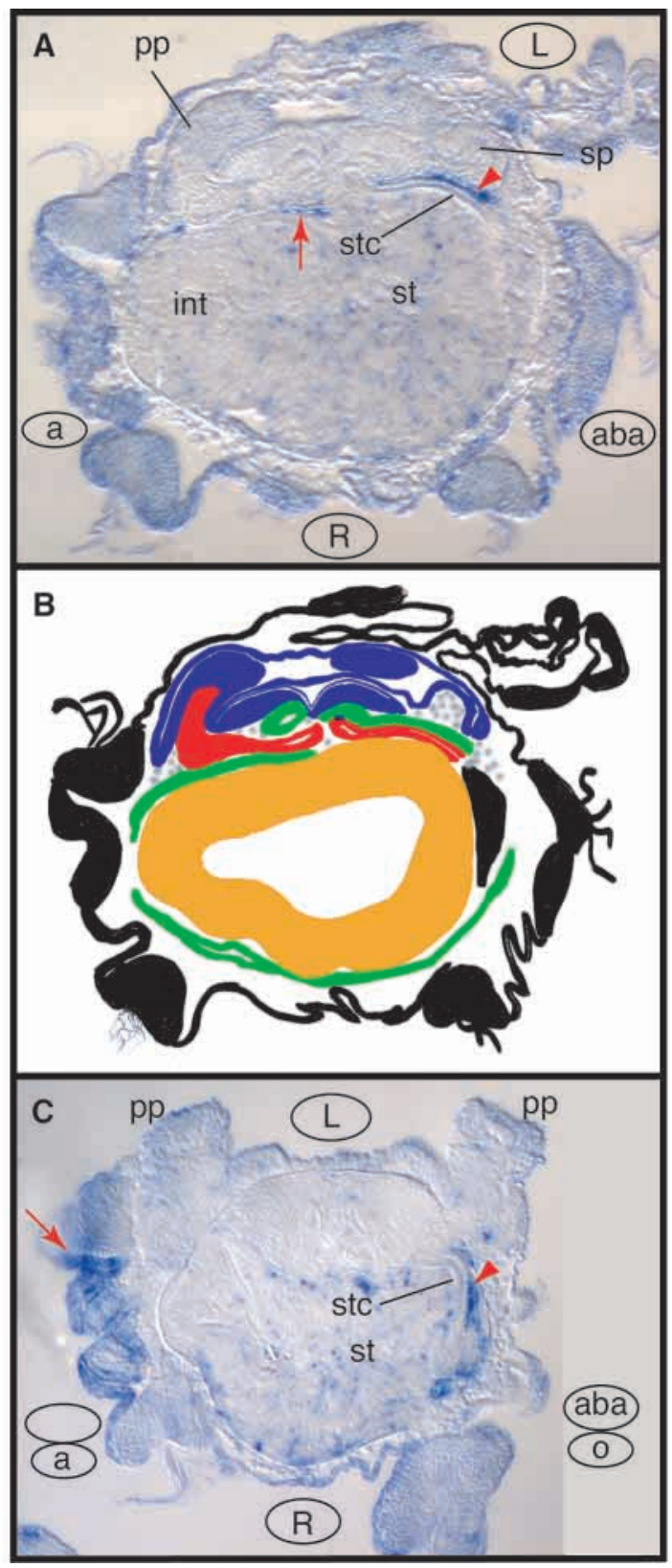

Fig. 4. Somatocoelar expression of $\mathrm{SpHox} 7$ and $\mathrm{SpHox} 8$ near the stone canal. (A) Transverse section stained for SpHox8. Expression is detected in the somatocoel alongside the stone canal (stc, arrowhead). Less signal is detected in somatocoelar regions located more central to the rudiment (arrow). (B) Diagram of the section in A. The future adult oral surface is oriented toward the top. Mesenchyme is indicated in gray. See Fig. 2 for color coding. (C) Oblique section transecting from the aboral-anal (vertex region, arrow) to the abanal-oral (stone canal region, arrowhead) sides of a metamorphosing animal in which the somatocoelar staining for SpHox7 is indicated. Note the persistence of the aboral ectoderm expression at the vertex (arrow). sp, adult spine; see legends to Figs 1 and 2 for other abbreviations

structure that connects the nascent water vascular system to the outside via the hydropore. Fig. 4 shows that expression of both the SpHox7 and SpHox8 genes is dramatically enhanced in the immediate vicinity of this structure. None of the five genes studied was expressed in the dental sacs, derivatives of the left somatocoel, where SpHox3 is used (Arenas-Mena et al., 1998).

\section{Hox gene expression outside the somatocoels}

The aspects of Hox gene expression so far considered are exclusively somatocoelar, i.e. exclusively mesodermal, and these are indeed the predominant (and for SpHox 8 and SpHox $9 / 10$ the only domains of detectable transcription (Table 1)). As already noted, however, SpHox 11/13b, the most $5^{\prime}$ of the genes studied, was also expressed in the endoderm, i.e. in the anal region and the distal part of the intestine during early rudiment stages (Fig. 2I,J). Restriction to the hindgut and anus is evident in early larvae (not shown), though this domain of endodermal expression fades out late in larval development. As summarized in Table 1, several kinds of developing peripheral structures that are destined to be carried forward into the adult stage also display expression of $S p H o x 11 / 13 \mathrm{~b}$ and SpHox 11/13a in the larva. These genes are used similarly to one another outside of the rudiment proper in many small structures, particularly in epithelial cells that are derived from the aboral ectoderm and are associated with forming test elements (Figs 3F, 5). Note that in the high-magnification images of Fig. 5B,F the expression of these two genes is confined to the epithelial rather than mesodermal aspects of these structures. Similarly, SpHox $11 / 13 b$ is shown in Fig. 5C to be expressed in tissue that lies adjacent to the nascent adult test plate, probably from its position in the larva the madreporic plate. Other epithelial derivatives of the oral ectoderm in which these two genes are used include the vestibular margin (Figs 5A,D,E, 3F; and ectoderm lateral to or covering spines (Fig. 5D,E). These domains of expression have two factors in common: they are all ectodermal and they also occur in the immediate vicinity of particular forming structures, generally endoskeletal components, i.e. spines and test plates. These endoskeletal structures are specific morphological features of echinoids, and expression of SpHoxl1/13a and SpHox11/13b within them would seem clear examples of evolutionary co-option.

Further such co-options are visible in the peripheral patterns of expression of SpHox7, which, as illustrated in Fig. 6 and Table 1, is the most diversely used of the genes studied. During embryogenesis $\mathrm{SpHox} 7$ is expressed in the vertex of the aboral ectoderm (Angerer et al., 1989), and this domain of expression persists far into larval development, as shown in Figs 6B,D, 4C. In the transverse sections in Fig. 6B,D, the intestine wall or surrounding mesenchyme can also be seen to express the SpHox7 gene (mesenchymal expression is also to be seen in Fig. 6F,E,H), while the adjacent somatocoels do not express this gene. In addition to these peripheral loci of expression, however, $\operatorname{SpHox} 7$ was also expressed within the rudiment, in a unique set of developing tissues, which change progressively. An interpretive diagram of relevant structures in the rudiment is shown in Fig. 6A and diagrams representing the section shown in Fig. 6B,F,H are given, respectively, in Fig. 6C,G,I. Two unidentified groups of cells on each side, lateral to forming podia (pp) at the base of the epineural folds expressed SpHox7, as shown in Fig. 6E. The SpHox7 gene expression patterns reported here could be or are associated with the radial central nervous system of the rudiment (see Fig. 6A). The radial nerve (yellow) arises at the base of a thickened domain of vestibular ectoderm (blue) derivatives, overlying the radial 
Fig. 5. Peripheral expression domains of SpHox1 1/13a and SpHox11/13b. (A) SpHox11/13b is expressed in a structure located in the larval right side which is associated with one of the primordia of the adult test plates (arrowhead). This gene is also expressed in regions of the larval epithelium associated with the vestibular margin (arrows). (B) Transcripts of SpHox11/13a appear in the epithelium of structures associated with nascent adult test plates (red arrowheads). (C) Detail of a structure located near the hydropore (arrowhead), which is the location of the prospective adult madreporic plate that expresses SpHox 11/13b; 1m, larval mouth. (D) Transverse
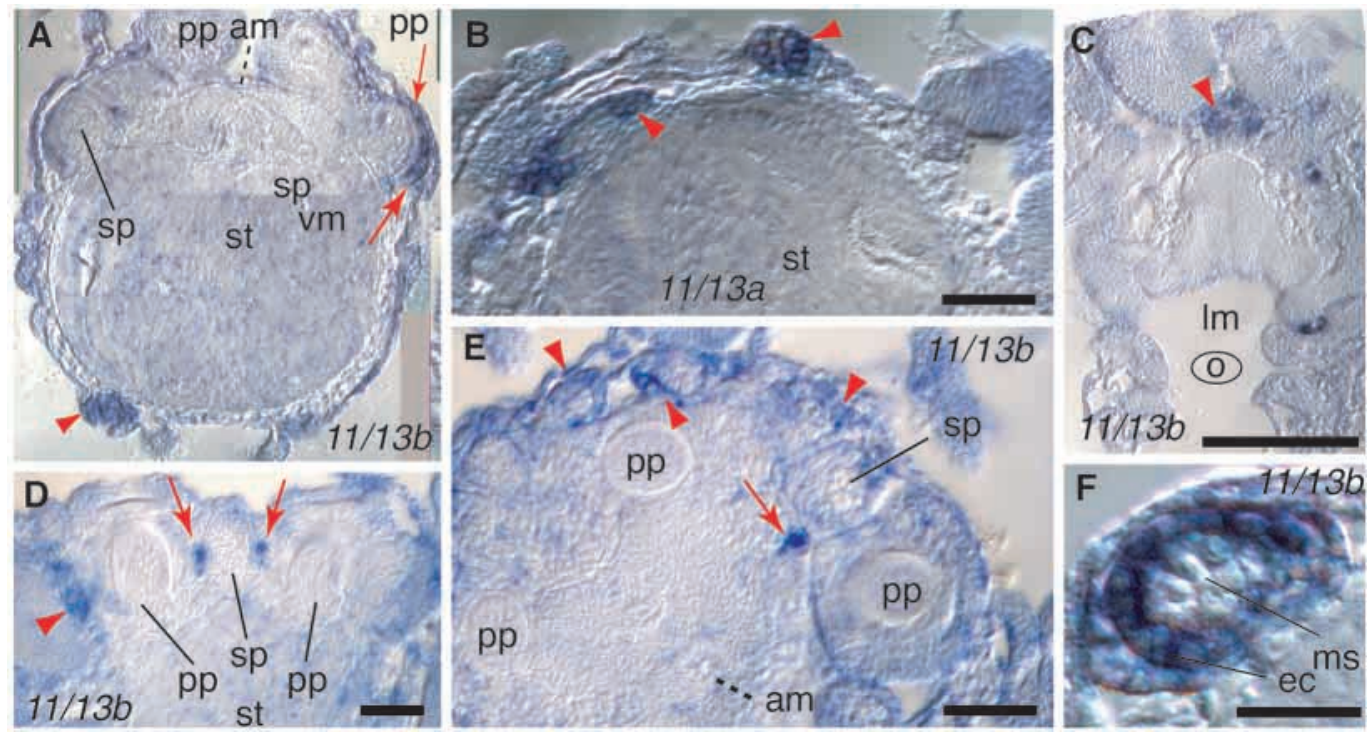
section of the rudiment displaying pattern of staining in ectodermal derivatives around a nascent spine (arrows), and in the periphery (arrowhead). (E) Section in the adult oral plane. Arrows and arrowheads as in D. (F) Detail illustrating that expression of SpHox11/13b is restricted to the ectoderm of the nascent structures of the plates. Other abbreviations as in previous Figs. Scale bars: $20 \mu \mathrm{m}$ in A-E; $10 \mu \mathrm{m}$ in F.

canal (rc). The epineural canal (epc, Fig. 6A) overlies the radial nerve and it is formed after the fusion of the epineural folds (ef, Fig. 7I). In Fig. 6F,G the staining at locus 3 may lie within the radial nerve precursor cells or is at least immediately adjacent to them. Locus 4 of Fig. 6F (cf. A) may represent the central nerve ring, or it could involve elements of dental sacs (ds), which are somatocoelar derivatives (green in Fig. 6A,G,I). Staining in or directly around the radial nerve is also seen in Fig. 6H, again at locus 3 (cf. Fig. 6A and 6I). Staining at locus 2 here indicates $\mathrm{SpHox} 7$ expression in the epineural folds during the formation of the epineural canal (Fig. 7H). Expression in locus 1 (Fig. 6E,F) is initially continuous with the expression in locus 2, and perhaps also with locus 3 . During the latest stages the expression in locus 1 becomes separated into domains that are pentamerally organized.

\section{DISCUSSION}

One way to summarize simply the complex patterns of posterior Hox gene expression described is to consider that they illustrate three separate issues. First, the developmental significance of SpHox11/13b expression in the hindgut; second, the many specific co-options to echinoderm-specific functions that are particularly evident for $\mathrm{SpHox7}$; and third, the spatially sequential and dynamic somatocoelar expression pattern in which all five of the genes studied are used.

SpHox11/13b is expressed intensively in the anal and adjacent hindgut endoderm of the larva, and, unlike its somatocoelar expression, this pattern of transcription is established far back in embryonic development (Dobias et al., 1996; C. A.-M., A. R. C. and E. H. D., unpublished). This can perhaps be understood in terms of a common feature of maximal indirect development: we pointed out earlier that while the mesodermal structures and central nervous systems of the adult body plan are always de novo products of the postembryonic developmental process in indirect development, the digestive tract or at least portions of it are often retained from embryogenesis (Peterson et al., 1997, 2000b). SpHoxl1/13b is the most $5^{\prime}$ of the Hox genes so far identified in the S. purpuratus Hox cluster (Martinez et al., 1999; however, additional posterior genes have been discovered in other echinoderms; Mito and Endo, 2000). Embryonic specification of the hindgut and anus apparently involves regional activation of $S p H o x 11 / 13 b$, just as posterior Hox genes are expressed in the hindgut of other animals. This gene simply continues to be expressed in the anus and hindgut throughout most of larval development in S. purpuratus, i.e. so long as these structures remain functional.

Co-option of regulatory genes to the specific purposes of echinoderm development has evidently been a major, or the major, process in the evolution of this clade (Lowe and Wray, 1997). We here add several apparent new examples. For instance, both SpHox11/13b and SpHox11/13a are expressed in the vicinity of new structures associated with skeletal plates (Fig. 5). The most remarkable cases are afforded by SpHox 7 (Fig. 6): this gene is expressed in many locations both within and peripheral to the rudiment, in ectodermal or epithelial components. SpHox7 is also used in the neuroectodermal tissues within which the pentameral ring nerve and the radial nerve system forms. This displays pentameral symmetry, by definition an echinoderm-specific character. The incidence of evolutionary co-option to clade-specific functions must be very great, considering how easy it is to find examples, that is, if one looks for it in organisms that differ in body plan from the arthropod and vertebrate examples that are most familiar to us (Lowe and Wray, 1997; this work).

\section{The somatocoelar 'Hox vector'}

SpHox7, SpHox8, SpHox9/10, SpHox11/13a and SpHox11/13b are all expressed in the somatocoelar mesoderm. The most unexpected and on the face of it puzzling aspect of our results is that their expression domains form a bilateral, sequential set of spatial patterns that are not oriented in accord with either 
Fig. 6. Multiple sites of $\mathrm{SpHox} 7$ expression. Loci of expression in early stages are shown in B,D. Later stages are depicted in E,F,H. (A) Diagram of an advanced rudiment (after Von Ubish, 1913; and MacBride, 1903). Color code as in Fig. 2: blue, vestibular ectodermal derivatives; green, somatocoel and dental sacs; yellow, elements of radial nerve and nerve ring; red, hydrocoel; orange, stomach and intestine; black, larval ectoderm and pharynx; gray, mesenchyme. Numbered labels indicate different loci of $\mathrm{SpHox} 7$ expression in the rudiment. Locus 1 is at the base of the epineural folds (ef) in the periphery of the rudiment; the broken lines indicate that the structures are behind the plane of the drawing, away from the reader. Locus 2 lies within the central domains of the epineural folds, in both upper and lower portions of the folds; in the diagram the folds are portrayed after they have united with one another (see explanation in I). Locus 3 is the internal wall of the epineural canal (epc), adjacent to the hydrocoel; it is coincident with the area where the cells of the radial nerve ( $\mathrm{rn}$ ) are formed. The region in direct contact with the epineural canal does not differentiate into neural tissue. Locus 4 refers to an area near the future mouth, where the nerve ring (nr) and the extensions from the dental sacs (ds, in green) are in close apposition. Other abbreviations as in earlier Figs. (B,C) Expression of SpHox7 in cells around the intestine (arrows; see also red arrowheads in E,F,H). These cells are not part of the adjacent somatocoel (s). They may be either mesenchyme or endoderm, though in F, arrowheads appear to indicate stained mesenchyme cells, for instance that denoted by the first arrowhead from the left. $\mathrm{SpHox} 7$ is also expressed in the aboral ectoderm of the larva shown in B (aec). (D) Transverse section at the level of the rudiment (see similar section in Fig. 2G for diagram). SpHox7 transcripts are detected in cells forming a ring around the distal constriction of the intestine (arrows). The staining is not found in the adjacent somatocoels (s). (E) Section at a peripheral region of the rudiment. Lateral to each primary podium, at the base of the epineural fold (ef) there are two groups of cells expressing SpHox7 (locus 1, cf. A); mesenchyme near the rudiment also expresses the gene (arrowhead, as in F,H). (F,G) Section through the center of a later rudiment stage, which cuts along a ray of the water vascular system, the progenitor of the radial canal (rc). There are cells expressing SpHox7 at the internal wall of the epineural canal (locus 3 in F,H), the same zone that generates the radial nerves (rn, in A,G). The expression does not extend to more peripheral regions (white arrowheads in F). Locus 4 indicates a region where the staining may be at the internal wall of the epineural canal, in the region of the future nerve ring (nr), or in extensions from the dental sacs, or both (see locus 4 in A). The locus 1 pattern is shown in a different orientation than in (E). (H,I) Section of an earlier stage than the one shown in F. The section cuts across a ray of the hydrocoel (rc). Cells at the base of the recently formed epineural canal express $\operatorname{SpHox} 7$ (locus 3 ). In I, the broken arrows indicate the morphogenetic progression of the epineural folds from the periphery towards the center of the rudiment. The epineural folds express SpHox7 (locus 2 in $\mathrm{H}$ ), in both the external and the internal sides of the fold. Scale bars represent $20 \mu \mathrm{m}$. Labels as in previous figures except aec, aboral ectoderm; ef, epinerural fold; epc, epineural canal; rc, radial canal; rn, radial nerve; vf, vestibular fold. the O/Abo axis of the larva or A/P axis of the adult (Peterson et al., 2000a). The somatocoelar expression pattern is in the plane of the flat, sac-like somatocoelar mesodermal sheets (see Fig. 2K,L). On the left-hand side this pattern is orthogonal to the nascent $\mathrm{A} / \mathrm{P}$ axis of the rudiment. But considered in light of the recent theory for the evolutionary origin of the echinoderm body plan put forth by Peterson et al. (2000a), this pattern turns out to be revealing rather than paradoxical.

In Fig. 7, we summarize the patterns of expression of the five posterior Hox genes in the somatocoelar tissues, both on 

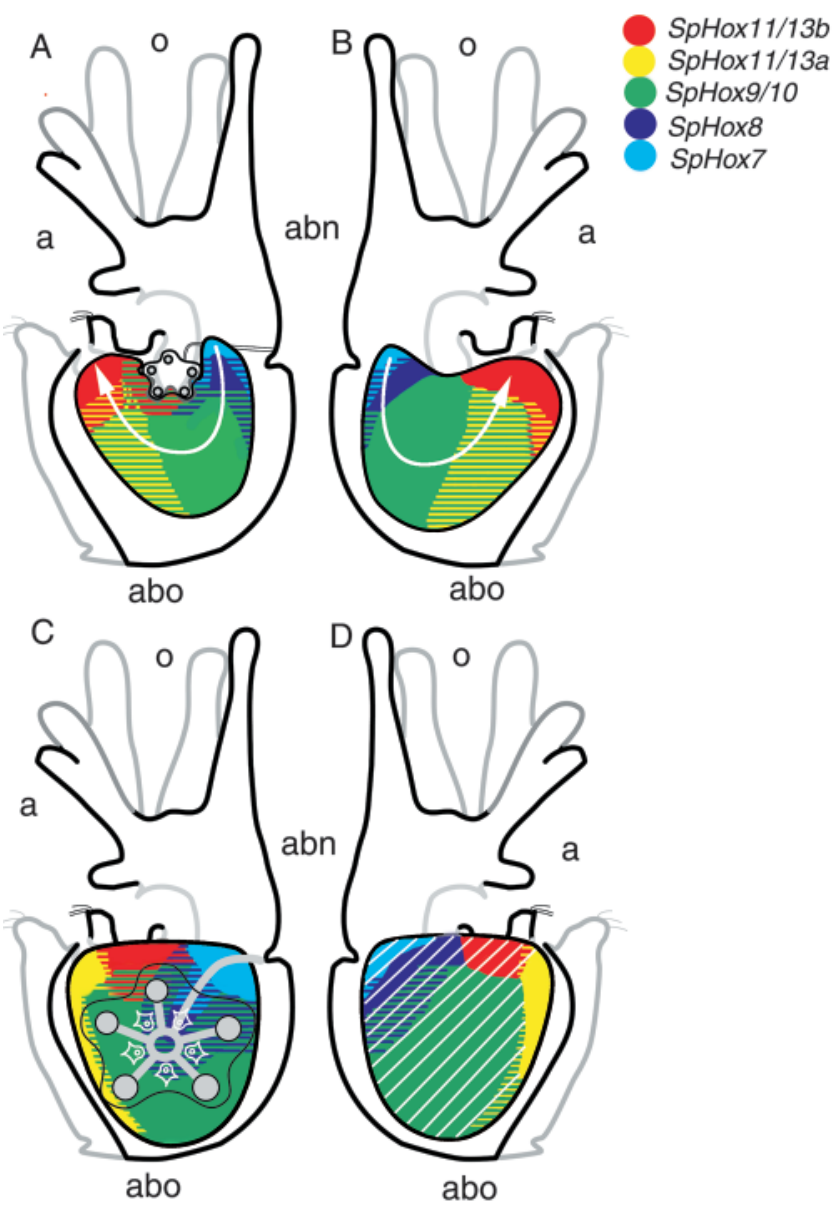

the right and left sides, and at early (Fig. 7A,B) and late (Fig. 7C,D) stages. Early on, i.e. at the stage when the pentameral organization of the rudiment has just become visible, the patterns of expression are similar on the two sides, except that on the left side (Fig. 7A) the expression domain of SpHox7 and SpHox8 extend from the abanal end of the somatocoel more centrad, towards the young rudiment, and likewise the SpHox11/13b expression domain extends more centrad from the anal end. This results in a more complex set of overlapping expression domains on the left side near the rudiment compared with those on the right-hand side (striped regions of Fig. 7A versus Fig. 7B). The result is that for about $120^{\circ}$ of its circumference, the rudiment confronts different combinations of Hox gene products in each quadrant. However, the rudiment develops in an independent pentamerally symmetrical manner; it is here that the fivefold organization of the echinoderm body plan is initially established. The adjacent tissues, including the digestive tract, skeletal elements and also the somatocoels, where the posterior Hox genes are expressed, display bilateral rather than pentameral organization. By late in rudiment development (Fig. 7C,D) right versus left asymmetries are much accentuated. For example, $\mathrm{SpHox} 7$ and SpHox8 expression display an asymmetrical stripe directly apposed to the position of the growing stone canal; these genes and SpHox9/10 now are expressed in somatocoelar mesoderm beneath the rudiment on the left-hand side; and in general most of the posterior Hox gene expression is lower on the right-hand side (white hatching in Fig. 7D), compared with the left.
Fig. 7. Summary of patterns of 'posterior' Hox gene expression in the somatocoels. Early stages are represented in A,B, and later stages in C,D. The color code (top right) represents the domains of expression of individual genes according to this work (Figs 2-4, and extensive additional data). Domains of overlapping gene expression are represented as bi-colored stripes. Each stage is shown from both left and right views to illustrate both somatocoels: $(A, C)$ left views, where pentaradial rudiment can be seen; (B,D) right views. The most $5^{\prime}$ gene of the cluster so far known, $S p H o x 11 / 13 b$, is expressed in the anal region and consecutively more 'anterior' genes are expressed in a sequential set of domains describing a curved pattern along the somatocoels (white arrows). The overlapping domains of expression are broader in more aboral regions. Except for SpHoxl 1/13a the gene expression domains are expanded or are stronger on the left side near the rudiment as development progresses (i.e. SpHox7, SpHox8, SpHox9/10 and SpHox1 1/13b; Fig. 3); compare C with D. In C, the water vascular system is indicated within the outline of the rudiment in gray, and the dental sacs in white. In D, the patterns of expression in the right somatocoel are similar to those in early stages shown in B, except for the absence of SpHox1 1/13b expression around the anus; the transcript levels also seem lower (indicated by oblique white lines), compared with those detected in the left somatocoel (C), except for SpHoxl 1/13a, which stains similarly in both somatocoels. The left somatocoel has now grown under the rudiment, and the expression domains of the Hox genes extend to this region as well. Besides the general enhancement affecting most Hox genes near the rudiment, there is a further enhancement of SpHox7 and SpHox8 expression around the stone canal (on the left side only, shown in C). No pentameral pattern of expression has been observed for any of the five 'posterior' genes in the somatocoel nor has expression been found in the dental sacs (depicted in white in C). Positional labels: a, anal; abn, abanal; abo, aboral; L, left; o, oral; R, right.

The major importance of Fig. 7 lies in the curved white arrows in the top pair of drawings. These illustrate the somatocoelar 'Hox vector', i.e. the orientation of the sequence of expression domains of genes located $3^{\prime}$ to $5^{\prime}$ in the Hox cluster. The sequence of expression domains is colinear with the order of the genes in the Hox cluster (Martinez et al., 1999). As the white arrows show, these sequential patterns extend continuously (SpHox7-SpHox11/13b) in an anal direction along a curved axis with respect to the morphology of the somatocoel that parallels the curvature of the digestive tract. Were the digestive tract and the accompanying somatocoels straightened out (see Fig. 2K,L), the arrangement of the Hox vector' would be linear instead of curved. Such a straight digestive tract is found in the very similar larva of the sister group Hemichordates (Peterson et al., 1997), where a linear Hox vector within the posterior coeloms would be predicted.

\section{Evolution of the echinoderm body plan}

The common ancestor of echinoderms and hemichordates was almost certainly a bilaterally organized animal in which the posterior pair of coeloms terminated near the anus. We see that all of the posterior Hox genes are in fact expressed in the somatocoels. However, the pattern shown in Fig. 7 enriches our image of the evolutionary transformations that generated the pentameral structure that has been characteristic of most adult echinoderm body plans ever since the Early Cambrian. The original $\mathrm{A} / \mathrm{P}$ orientation of the somatocoels that can safely be inferred for the bilateral common ancestor of echinoderms and hemichordates can still be seen in hemichordates. In the 
evolutionary process leading to $S$. purpuratus this axis was evidently altered by a $90^{\circ}$ shift in the digestive tract and the associated somatocoelar structures, so that what was originally at the tail of the animal is now on one side (thus, presumes or rather predicts, that in hemichordates the sequence of homologous Hox gene expression patterns will run directly along the A/P body axis). Part of the function served thereby is implied by the patterns of Fig. 7C,D. The definitive morphological change in the evolution of the echinoderm body plan is coelomic stacking (Peterson et al., 2000a) such that viewed from the oral surface of the pentaradial adult body plan (as in Fig. 7C), the left somatocoel comes to lie beneath (actually interdigitated within) the hydrocoel of the rudiment; further below the plane of the page, the right somatocoel comes to lie beneath the left. The roughly circular shape the somatocoels assume as they surround the digestive tract late in development, including its stubby intestine, facilitates this arrangement: were the gut removed, the coeloms would resemble a stack of coins. The morphological stacking transformation would be much more different to conceive were the gut and somatocoels elongate linear structures. However, the price that had to be paid is also clear from Fig. 7: formation of the adult body plan requires formation of a new terminal hind gut and anus, as the original one (colored red for the original domain of Hox $11 / 13 \mathrm{~b}$ in Fig. 7A,B) is in the wrong place. Indeed, in echinoids, neither the larval hindgut and anus nor the esophagus survives metamorphosis. These are replaced in the juvenile during the period between metamorphosis and the resumption of feeding. We do not yet know whether the posterior Hox genes are reactivated during this process.

The difference between the right and left somatocoelar Hox gene expression patterns shown in Fig. 7C,D, both quantitative and qualitative, can be regarded as molecular correlates of the developmental process of coelomic stacking. Thus, the left somatocoel, which is in contact with the rudiment and forms parts of the oral region of the animal (e.g. its dental sacs; Fig. 6), expresses the Hox genes differently than does the right somatocoel. The right somatocoel contributes to the development of some of the structures of the lateral and anal surfaces of the adult, and correlated with its different developmental role is a different and less intense pattern of posterior Hox gene expression. Unfortunately, very little is known about the developmental biology of adult structures in the late larva, e.g. the specific contribution of the coeloms to the mesenteries, the gonad rudiment or the complex and regionally specific endoskeletal plates and spines. If the Hox gene expression patterns of the right somatocoel indeed play a role in setting up spatial progenitor fields in which subsequent morphogenetic events are to occur, these would be among the likely body parts for which they might be required.

\section{Predominance of mesodermal expression}

For the Hox genes studied, the somatocoels are the major loci of expression of the four more posterior, as only SpHox7 displays extensive expression within the rudiment (Fig. 6). Thus, as we have seen, it is expressed in epithelial and neuroectodermal tissues. SpHoxl1/13b also plays multiple roles, being expressed in the hindgut and anal endoderm, as well as in the adjacent somatocoelar domains. But SpHox9/10 and SpHox 8 are expressed exclusively in the somatocoels. Given the highly organized pattern shown in Fig. 7, somatocoelar expression certainly represents the most basal of all of the posterior Hox gene functions during body plan development in $S$. purpuratus, except perhaps for the expression of SpHox11/13b, which in its remote ancestors marked the posterior terminus of the animal. This is to say that the most important and perhaps the most ancient developmental functions of these posterior Hox genes in this clade of animals are played out in mesodermal tissues. There is no detectable expression of SpHox8-SpHox11/13b in the progenitor field of the radial central nervous system of the adult body plan, while in both vertebrates (see review by McGinnis and Krumlauf, 1992) and invertebrate chordates (Wada et al., 1999) the cognate Hox genes are expressed in thoracic and posterior regions of the dorsal nervous system and in mesodermal derivatives. It will be interesting to determine whether these same genes are expressed in the dorsal (or ventral) nerve cords of developing hemichordates; if not, this co-option to CNS patterning of posterior Hox genes in chordate ancestors may have been an important specific aspect of chordate evolution. Since the hemichordates are the echinoderm sister group we predict that the posterior Hox genes of enteropneust hemichordates will be expressed in an $\mathrm{A} / \mathrm{P}$ series in the metacoels, but without leftright asymmetry. In any case our observations do not sit well, i.e. parsimoniously, with the theory that the original, pleisiomorphic role of Hox genes in evolution was patterning of the A/P dimension of the central nervous system (Wada et al., 1999). As we have argued elsewhere (Davidson et al., 1995; Peterson and Davidson, 2000; Peterson et al., 2000b), a fundamental and novel event in bilaterian evolution must have been the appearance of mechanisms for patterning threedimensional mesodermal structures. The creation of regulatory networks controlling regional specification of the mesoderm, which included genes of the Hox cluster, could have constituted a key step in this process.

We thank Dr Kevin Peterson for his extremely helpful review. This work was supported by the Stowers Institute for Medical Research; the National Science Foundation, Developmental Mechanism Program, Grant No. IBN-9604454; and the Fundamental Biology Research Program of the Life Sciences Division of the National Aeronautics and Space Administration/ Ames Grant NAG2-1368.

\section{REFERENCES}

Angerer, L. M., Dolecki, G. J., Gagnon, M., Lum, R., Wang, G., Yang, Q., Humphreys, T. and Angerer, R. C. (1989). Progressively restricted expressionof a homeobox gene within the aboral ectoderm of developing sea urchin embryos. Genes Dev. 3, 370-382.

Arenas-Mena, C., Martinez, P., Cameron, R. A. and Davidson, E. H. (1998). Expression of the Hox gene complex in the indirect development of a sea urchin. Proc. Natl. Acad. Sci. USA 95, 13062-13067.

Bromham, L.D. and Degnan, B. M. (1999). Hemichordates and deuterostome evolution: Robust molecular phylogenetic support for a hemichordate plus echinoderm clade. Evol. Dev. 1, 166-171.

Cameron, C. B., Garey, J. R. and Swalla, B. J. (2000). Evolution of the chordate body plan: new insights from phylogenetic analyses of deuterostome phyla. Proc. Natl. Acad. Sci. USA 97, 4469-4474.

Cameron, R. A., Britten, R. J. and Davidson, E. H. (1989). Expression of two actin genes during larval development in the sea urchin Strongylocentrotus purpuratus. Mol. Reprod. Dev. 1, 149-155.

Cameron, R. A., Mahairas, G., Rast, J. P., Martinez, P., Biondi, T. R., Swartzell, S., Wallace, J. C., Poustka, A. J., Livingston, B. T., Wray, G. 
A. et al. (2000). A sea urchin genome project: Sequence scan, virtual map, and additional resources. Proc. Natl. Acad. Sci. USA 97, 9514-9518.

Davidson, E. H., Peterson, K. and Cameron, R. A. (1995). Origin of the adult bilaterian body plans: Evolution of developmental regulatory mechanisms. Science 270, 1319-1325.

Davidson, E. H., Cameron, R. A. and Ransick, A. (1998). Specification of cell fate in the sea urchin embryo: Summary and some proposed mechanisms. Development 125, 3269-3290.

Dobias, S. L., Zhao, A. Z., Tan, H., Bell, J. R. and Maxson, R. (1996). SpHbox7, a new Abd-B class homeobox gene from the sea urchin Strongylocentrotus purpuratus: insights into the evolution of Hox gene expression and funciton. Dev. Dyn. 207, 450-460.

Hyman, L. H. (1955). The Invertebrates, Vol. IV, Echinodermata. New York: McGraw-Hill.

Lowe, C. J. and Wray, G. A. (1997). Radical alterations in the roles of homeobox genes during echinoderm evolution. Nature 389, 718-721.

Martinez, P., Rast, J. P., Arenas-Mena, C. and Davidson, E. H. (1999). Organization of an echinoderm Hox gene cluster. Proc. Natl. Acad. Sci. USA 96, 1469-1474, 1999.

MacBride, E. (1903). The development of Echinus esculentus, together with some points on the development of E. miliaris and E. acutus. Philos. Trans. R. Soc. London Ser. B 195, 285-330.

McGinnis, W. and Krumlauf, R. (1992). Homeobox genes and axial patterning. Cell 68, 283-302.

Metschnikoff, E. (1881). Über die systematische Stellung von Balanoglassus. Zool. Anz. 4.

Mito, T. and Endo, K. (2000). PCR survey of Hox genes in the crinoid and ophiuroid: Evidence for anterior conservation and posterior expansion in the echinoderm hox gene cluster. Mol. Phylogenet. Evol. 14, 75-388.

Mooi, R. and David, B. (1997). Skeletal homologies in echinoderms. Paleontol. Soc. Pap. 3, 305-335.

Pearse, J. S. and Cameron, R. A. (1991). Echinodermata: Echinoidea. In Reproduction of Marine Invertebrates, Vol. VI, Echonoderms and Lophophorates (ed. A. C. Giese, J. S. Pearse and V. B. Pears), pp. 513-622. Pacific Grove, CA: Boxwood.

Peterson, K. J. and Davidson, E. H. (2000). Regulatory evolution and the origin of the bilaterians. Proc. Natl. Acad. Sci. USA 97, 4430-4433.

Peterson, K. J., Cameron, R. A. and Davidson, E. H. (1997). Set-aside cells in maximal indirect development: evolutionary and developmental significance. BioEssays 19, 623-631.

Peterson, K. J., Arenas-Mena, C. and Davidson, E. H. (2000a). The A/P axis in echinoderm ontogeny and evolution: Evidence from fossils and molecules. Evol. Dev. 2, 93-101.

Peterson, K. J., Cameron, R. A. and Davidson, E. H. (2000b). Bilaterian origins: significance of new experimental observations. Dev. Biol. 219, 117.

Von Ubisch, L. (1913). Die Entwicklung von Strongylocentrotus lividus. Z Wiss Zool. 106, 409-448.

Wada, H. and Satoh, N. (1994). Details of the evolutionary history from invertebrates to vertebrates, as deduced from the sequences of 18S rDNA. Proc. Natl. Acad. Sci. USA 91, 1801-1804.

Wada, H., Garcia-Fernandez, J. and Holland, P. W. H. (1999). Colinear and segmental expression of amphioxus Hox genes. Dev. Biol. 213, $131-141$. 